\title{
Hund und Katze zur Allergieprävention
}

Hier steht eine Anzeige.
Obwohl Haustiere eine Hauptquelle für Innenraumallergene sind, scheinen sie die Entstehung von allergischer Rhinitis und Asthma nicht zu fördern. Eher könnte das Gegenteil zutreffen, wie aktuelle Studienergebnisse zeigen.

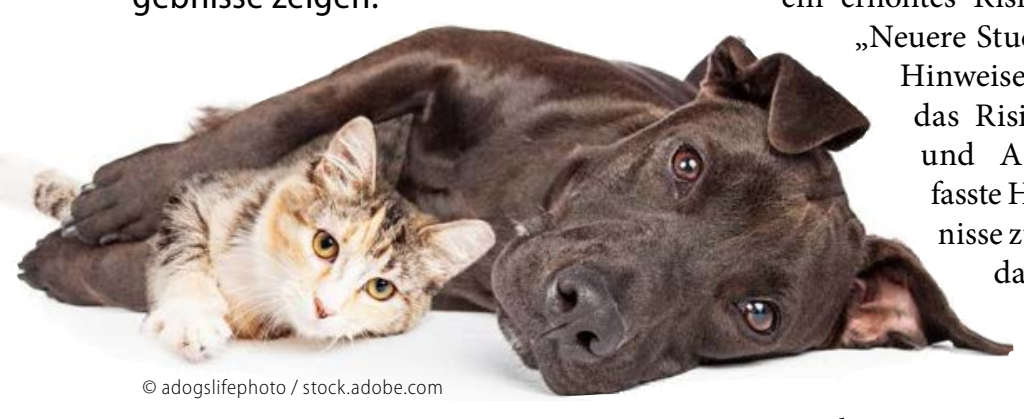

Stadtkinder, die mit Katze oder Hund groß wurden, hatten in sechs von neun Longitudinalstudien ein vermindertes Allergierisiko. Nur in einer ergab sich kein Zusammenhang und in zweien war für Kinder mit familiärer Vorbelastung ein erhöhtes Risiko zu erkennen. „Neuere Studien liefern keine Risiko für Allergien Asthma erhöhen", fasste Heinrich die Ergebnisse zusammen. Es gebe daher keine Rechtfertigung, zum Zwecke der Prävention von Haustieren abzuraten. Auch die Leitlinie Allergieprävention sieht für Kinder ohne atopische Familienanamnese keine Einschränkungen vor. Bei vorbelasteten Kindern empfiehlt sie dagegen, auf die Anschaffung einer Katze zu verzichten.

Laut Heinrich werden durch die Studien sogar eher protektive Effekte von Haustieren, insbesondere von Hunden, nahegelegt. Eine reverse Kausalität - weil es in einer Familie bereits Allergien gibt, legt sie sich kein Haustier zu - scheint zwar bei Katzen eine Rolle zu spielen, bei Hunden allerdings nicht. Dass die Tiere eine Immuntoleranz induzieren, hält Heinrich für „unwahrscheinlich“. Er vermutet eher einen Zusammenhang mit der Exposition gegenüber anderen biologischen Kontaminationen, die von den Tieren ins Haus gebracht werden. Letztlich sei der Mechanismus aber bislang nicht geklärt. „Solange das nicht der Fall ist, kann die Haustierhaltung auch nicht zur Allergieprävention empfohlen werden“, so sein Fazit.

Dr. Beate Schumacher

basierend auf: Heinrich J: Allergie gegen Tiere: Alleine leben oder im Privatzoo? Session: Allergieprävention: Karenz oder Exposition? 58. Kongress der Deutschen Gesellschaft für Pneumologie und Beatmungsmedizin. Stuttgart. 24. März 2017

1. Lødrup et al. PLOS One 2012

2. Collin et al. Clin Exp Allerg 2015

3. Lodge et al. Clin Dev Immunol 2012 